\title{
TIME DEPENDENT TUNNELING IN DOUBLE-BARRIER ZnSe/ZnTe STRUCTURES*
}

\author{
P. Bala, J.S. KWiatKowski and W. Bala \\ Institute of Physics, N. Copernicus University, Grudziądzka 5, 87-100 Toruń, Poland
}

\begin{abstract}
The wave-packet time dependent quantum mechanics is used to calculate tunneling probability through a double-barrier $\mathrm{ZnSe} / \mathrm{ZnTe}$ structure. The time dependent $I-V$ characteristics are obtained for several structures. The resonant peaks are observed and their changes with the barrier parameters are monitored.
\end{abstract}

PACS numbers: 03.65.-w, 73.40.Gk, 73.60.Fw

Recently there has been great both experimental [1] and theoretical interest [2] in quantum tunneling devices. However, the dynamical aspects of tunneling, although important for the physical understanding as well as for applications, have remained largely unclear [3]. Most theoretical works concerning resonant tunneling involve time independent methods such as finding eigenvalues for quantum wells and superlattice structures. The transmission probability for such structures is usually calculated using plane wave approximation and this gives no information about time resolution of tunneling process. Recent experimental techniques allow measurement of the tunneling time in double quantum wells and, thus, provide information about maximum switching frequency of such devices [4].

In this paper we deal with a time dependent quantum mechanics of a wave packet to calculate the tunneling probability for a double-barrier structure $n$-type $\mathrm{ZnSe} / p$-type $\mathrm{ZnTe}$. The energy diagram of the structure is presented in the insert in Fig. 1. The electron is treated in full quantum mechanical way within the effective mass approximation. The detailed electron dynamics is obtained by solving the time dependent Schrödinger equation

$$
\mathrm{i} \hbar \frac{\partial \Psi(x, t)}{\partial t}=\mathcal{H} \Psi(x, t) .
$$

In particular case, a powerful numerical algorithm based on the Chebychev polynomial expansion [5-7] is used. The wave function $\Psi(t+\Delta t)$ represented over a discrete grid after a small time step $\Delta t$ can be approximated as

$$
\Psi(t+\Delta t) \cong \mathrm{c}^{-\mathrm{i}\left(\Delta E / 2+V_{\min }\right) \Delta t / \hbar} \sum_{m=0}^{M} a_{m}\left(\frac{\Delta E \Delta t}{2 \hbar}\right) \Phi_{m}\left(-\mathrm{i} \mathcal{H}_{\text {norm }}\right) \Psi(t),
$$

${ }^{*}$ This work is supported in part by the Committee for Scientific Research under grant No. 203299101. 


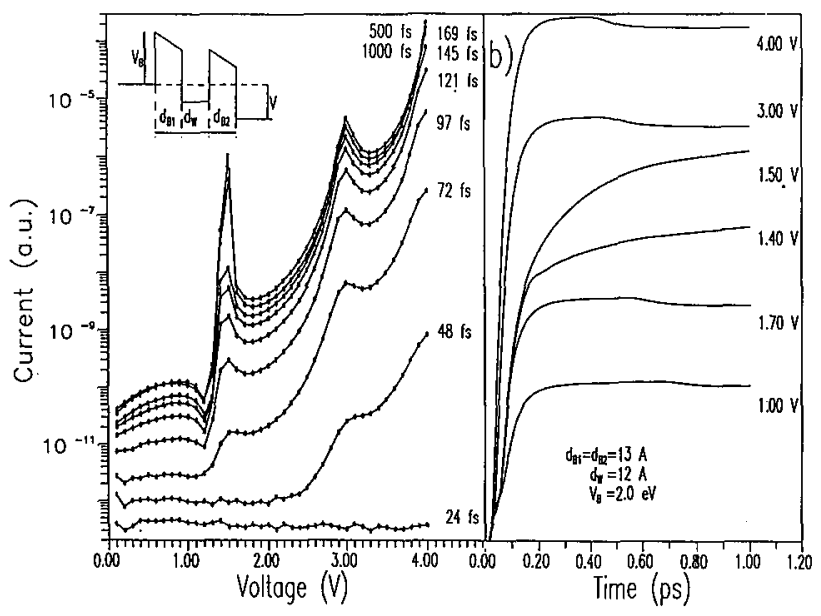

Fig. 1. $I-V$ characteristics at different times (a) and time dependence of tunneling current at different applied voltages (b) for the double-barrier $\mathrm{ZnTe} / \mathrm{ZnSe} / \mathrm{ZnTe}$ structure with dimensions $d_{\mathrm{W}}=12 \AA, d_{\mathrm{B} 1}=d_{\mathrm{B} 2}=13 \AA$ and $V_{\mathrm{B}}=2.0 \mathrm{eV}$. The insert presents the energy diagram for double-barrier structure with applied voltage $V$.

where $\mathcal{H}_{\text {norm }}$ is the Hamiltonian normalized to the energy range $(-1,1), V_{\min }$ and $\Delta E$ are energy minimum and energy range of Hamiltonian over the grid $[5,6]$. The expansion coefficients $a_{m}$ can be expressed in terms of Bessel functions $J_{m}$. The complex Chebychev polynomials $\Phi_{m}$ are calculated using recurrence formulas [6]:

$$
\Phi_{0}(X)=I, \quad \Phi_{1}(X)=X, \quad \Phi_{m}(X)=X \Phi_{m-1}(X)-\Phi_{m}(X) .
$$

The grid size (2048 points, distance between points $2.2 \AA)$, the time step ( $\Delta t=$ $1.0 \mathrm{fs})$ and the number of polynomials $(M=30) \mathrm{kept}$ in the expansion (1.2) are chosen in such a way to minimize the numerical errors [5]. The initial wave function is chosen to be Gaussian type with the width $150 \AA$ centered at $300 \AA$ to the left from the structure. Its momentum compares to this of the thermal motion at $300 \mathrm{~K}$.

The tunneling probability versus the voltage applied to the structure, i.e. the $I-V$ characteristics, is presented for different times in Fig. 1a. The resonant tunneling peaks and their time evolution can be well observed. In Fig. 1b the detailed time dependence of the tunneling probability is shown for different applied voltages. The tunneling process is quite rapid and after only $0.1 \mathrm{ps}$ the probability of finding the electron to the right from the structure remains constant. Quite different behavior is observed for voltages corresponding to the resonant tunneling conditions. In this case increase in the probability, after the first rapid period, is slower and reaches a stable value after a few picoseconds. It is caused by the trapping of the electron by the resonant level in the the potential well of the double-barrier structure. The probability of the tunneling to the resonant level is much higher (by approximately 2 orders of magnitude) than in nonreso- 
nant conditions and renders a significant probability of finding the electron in the potential well. The lifetime of the electron at the resonant level in the potential well is equal to only few picoseconds. This determines the time of the changes of the probability of finding the electron on the right hand side of the structure for voltages corresponding to the resonant conditions. In other cases the electron is not trapped in the well, because its energy differs from the energy of the levels in the potential well and the tunneling is in consequence faster, but it occurs with a much smaller probability.

In Fig. 2 the tunneling probability vs. applied voltage is presented for different double-barrier structures. The change of the position of resonant peaks with varying width of the potential well is quite strong and consistent with previous theoretical expectations. It is interesting to mention that at the voltage equal to

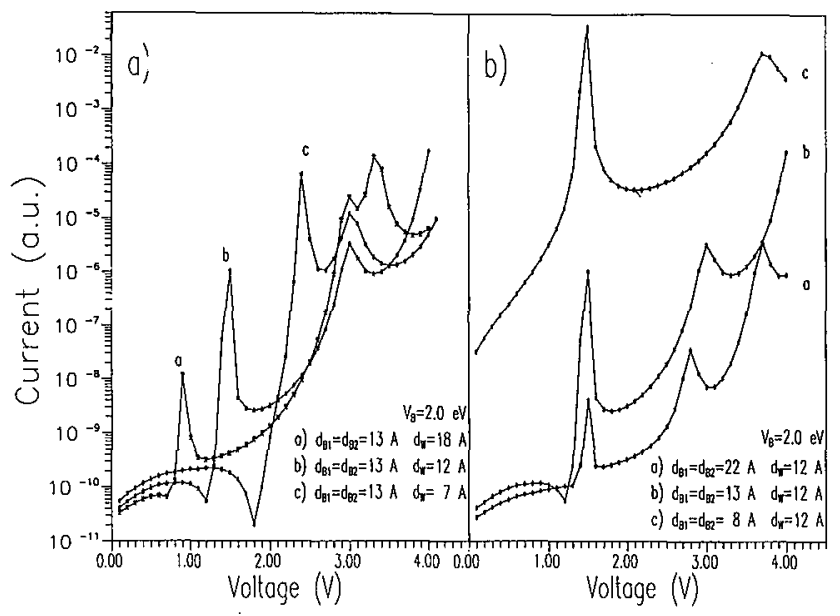

Fig. 2. $I-V$ characteristics at time 1.0 ps for double-barrier $\mathrm{ZnTe} / \mathrm{ZnSe} / \mathrm{ZnTe}$ structure with different potential well (a) and barrier (b) length. The values of well width $d_{\mathrm{w}}$ and barrier widths $d_{\mathrm{B} 1}$ and $d_{\mathrm{B} 2}$ are also presented.

$3.0 \mathrm{~V}$, which corresponds to energy level of value $1.5 \mathrm{eV}$ according to the bottom of the well of total depth $2.0 \mathrm{eV}$, the peak which does not depend on the well width is also observed. This phenomenon was observed in the experiments, but its origin was not clarified [8]. From our data presented in Fig. 2b it becomes clear that the position of this peak depends strongly on barrier width, but its origin is still unclear and should be a subject of further detailed study.

\section{References}

[1] Y.G. Gobato, J.M. Berroir, Y. Guldner, J.P. Vieren, F. Chevoir, B. Vinter, Phys. Rev. B 44, 13795 (1991).

[2] II. Young-Zhen, W. Chi-Ming, Appl. Phys. A 54, 191 (1992).

[3] E.H. Hauge, J.A. Stovneng, Rev. Mod. Phys. 61, 917 (1989). 
[4] M.G.W. Alexander, M. Nido, W.W. Ruhle, K. Kohler, Phys. Rev. B. 41, 12295 (1990).

[5] T.N. Truong, J.J. Tanner, P. Bala, J.A. McCammon, D.J. Kouri, B. Lesyng, D.K. Hoffman, J. Chem. Phys. 96, 2077 (1992).

[6] H. Tal-Ezer, R. Kosloff, J. Chem. Phys. 81, 3967 (1984).

[7] R. Kosloff, H. Tal-Ezer, Chem. Phys. Lett. 127, 223 (1981).

[8] M.W. Dellow, P.H. Beton, C.J.G.M. Lengerak, T.J. Foster, P.C. Main, L. Eaves, M. Henini, S.P. Beaumont, C.D.W. Wilkinson, Phys. Rev. Lett. 68, 1754 (1992). 